EPJ Web of Conferences 45, 01129 (2013)

DOI: $10.1051 /$ epjconf/20134501129

(C) Owned by the authors, published by EDP Sciences, 2013

\title{
Flow pulsation in the near-wall layer of impinging jets
}

\author{
V. Tesar̆ ${ }^{1}$ \\ ${ }^{1}$ Institute of Thermomechanics v.v.i., Academy of Sciences of the Czech Republic, Praha, Czech Republic
}

\begin{abstract}
Pulsation of impinging jets promises to become a useful way towards achieving the highest possible rate of passive scalar convective transport between fluid and a wall. Author investigated experimentally steady and pulsated impingement by hot-wire anemometer traversing along a radial line at a small height above the impingement wall. The data have shown two conspicuous local maxima of fluctuation intensity. In an attempt to reach understanding of these phenomena, numerical flowfield computations were also made, fitted to the experimental conditions. Despite simplification (isotropic handling of unsteadiness, eddies computed as Reynolds-type phase averages) the synergetic approach (experiment \& computation) revealed interesting correlation and resulted in useful interpretations of the old problem of the off-axis extremes - and also brings new views on their behaviour in the pulsating jet case.
\end{abstract}

\section{Introduction}

\subsection{Impinging jets}

Fluid jets impinging upon a solid wall are themselves a very interesting case of fluid flow, possessing a number of intriguing features that deserve closer studies. At the same time, they are important from the point of view of engineering applications, since they have the unique position of making possible the highest known intensity of convective heat and/or mass transfer between a fluid and a solid object. In view of this fact, they became an object of investigations already quite a long time ago in a number of research institutions around the world including the former Czechoslovakia, where impinging flows were since 1970s the subject of intensive experimental studies in SVÚSS Běchovice, a research institution which at that time was of high renomé. The primary aim of the research there, conducted in particular by $\mathrm{M}$. Korger and F. Křǐžek, were acquisition of data needed for applications in industrial processes - mainly drying textile and other flat materials, an operation necessarily involved in the processes of their manufacture. The interest naturally concentrated on the effects that took place on the impingement wall - for which was there developed the remarkable naphtalene sublimation method used successfully up to current days by Dr. Z. Trávníček in the Institute of Thermomechanics AS ČR, e.g. [31]. Another well-known centre where much information about the drying and heat transfer was accumulated was then the A. V. Luikov Heat and Mass Transfer Institute (Minsk, in present-day Belarus). An outstanding reference summarising almost all known at that time (especially the less easily accessible papers by the then Soviet authors) has been available since the 1980s as the monograph [1]. In general, less attention was at that time paid to the fluid mechanics of the impinging jet flows (much more complex than is generally believed) - perhaps because of the then politically determined inaccessibility of high quality anemometric instrumentation, without which it is difficult to study the jet flows with their typical unsteady vortical structures. A number of phenomena thus remained not understood. Among them, an important question was posed by existence of off-axis secondary maxima of convective transfer on the impingement wall. These are found around the primary central maximum in configurations with small nozzle distances $\mathrm{h}$ (general rule is they are present at about $\mathrm{h} / \mathrm{d} \leq 2$ ). The phenomenon was already discussed in 1965 by Gardon and Akfirat [25] who attributed it to transition into turbulence. Very soon thereafter this explanation proved to be wrong (by considering the responses to varied Reynolds number) so that the effect remained for a long time unexplained. Towards the end of the last century enough information was accumulated that it became obvious that the effect is associated with streamwise convected vortical organised motions in the jet [15]. Details, however, were and still remain not completely clear. The character of the flowfield has been inferred indirectly, from the patterns in visualised distributions on the impingement wall mainly of heat transfer using the infrared thermography [26] or thermochromic liquid crystals [27]. A study of the flowfield became available in [28], the drawback there being the validity for only very small Reynolds numbers $(\operatorname{Re}=500)$. The very recent surveys $[29,30]$ still base their explanations on conjectural hand-drawn pictures. In fact, ref. [30] speaks about "sparse literature on the subject". Of course, all the available data so far are valid only for the steady impingement. 


\subsection{Pulsatile impinging jets}

After the changes of the political system in this country, the present author received in 1995 a financial support for his research, in particular the grant Nr. 101/95/1421 from GAČR supporting the project "Transfer phenomena on a wall exposed to a fluid jet". In this project, a considerable part of effort was aimed at the problems of impinging jets. The grant made possible acquisition of a CTA hotwire anemometer (albeit only a single-channel version, at the bottom end of the cost scale) and also enabled buying components for setting up a simple automatic traversing gear. Because the outstanding feature of impinging jets is their exceptional high convection-transfer capability, it was natural to focus the interest on identifying the factors that limited further increase towards even higher values. It became gradually recognised that the limitation is due to the presence of a thin fluid layer - roughly corresponding to the viscous sublayer - of the nearly stagnant fluid held on the surface by viscous forces. This layer acts as a thermal insulator. The heat or mass transport has to cross this layer by conduction, a process much less effective (by several orders of magnitude in the case of gas) than convection.

One possibility how to remove this main obstacle is applying pulsation to demolish the stagnant layer. This may be done by periodic motion of the wall or of the nozzle from which the impinging jet issues - or by pulsation of the supplied fluid flow rate. It has been known since the Stokes' early investigation of unsteady boundary layer (on a highly simplified model representing astronomic clock pendulum [2]) that the thickness of the stagnant fluid layer (and hence its resistance to conduction transport) increases with increasing time after the fluid is set into motion. The increase in thickness is the effect of viscous momentum transfer action, diffusing away from the wall. In the oscillatory regime the thickness growth begins anew at

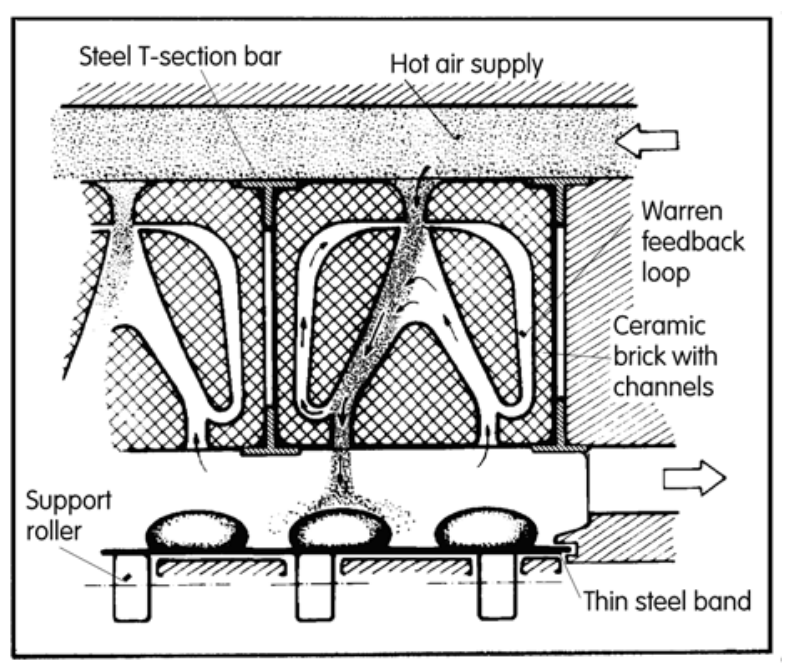

Fig 1 Example illustration from Patent [5]: heat transfer from hot air flows into baked food products is enhanced by pulsating jet impingement. Fluidic oscillators are made as depression channels on the surface of ceramic bricks. The idea of impinging jets and pulsation was much later successfully developed as described in [6].

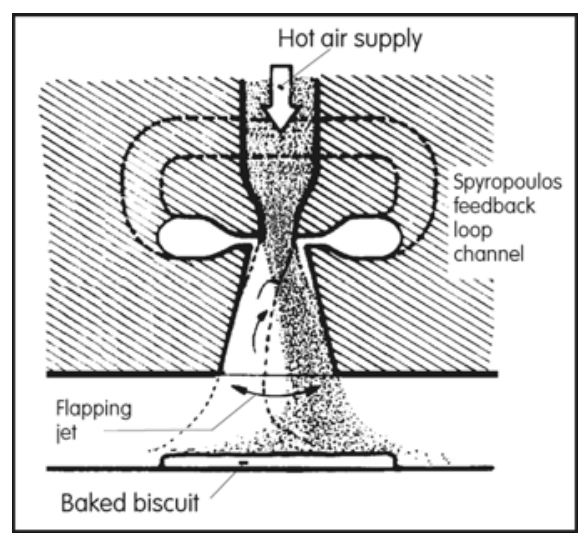

Fig 2 Another illustration from the Patent [5]: enhancement of heat transfer into the food products by impinging hot air jets flapped sideways. Oscillator uses a different type of feedback loop (connecting the two control nozzles) and the jet oscillates transversally rather than axially.

the beginning of each new period of the motion. Thus the time-mean thickness decreases and the resistance to conductive transport is lower.

That this is a sound idea and that heat or mass transfer is indeed increased by the pulsation was quite early verified by experiments. Martinelli and Boelter reported already in 1938 [3] an enhancement - as much as fivefold - of the convective heat transfer from a cylinder in fluid flow by vibrating it (this particularly strong effect was due to the oscillation interacting with the vortex shedding). Unfortunately, practical engineering use of the pulsation idea was disappointing. Natural choice of $20^{\text {th }}$ century engineers how to generate the pulsation was to design for the purpose a piston unit driven by an electric motor through speed-reducing gears - or perhaps a similarly driven turning-down valve. Such a unit, of course, is much more expensive and much more prone to malfunctions (and hence need of maintenance) than the

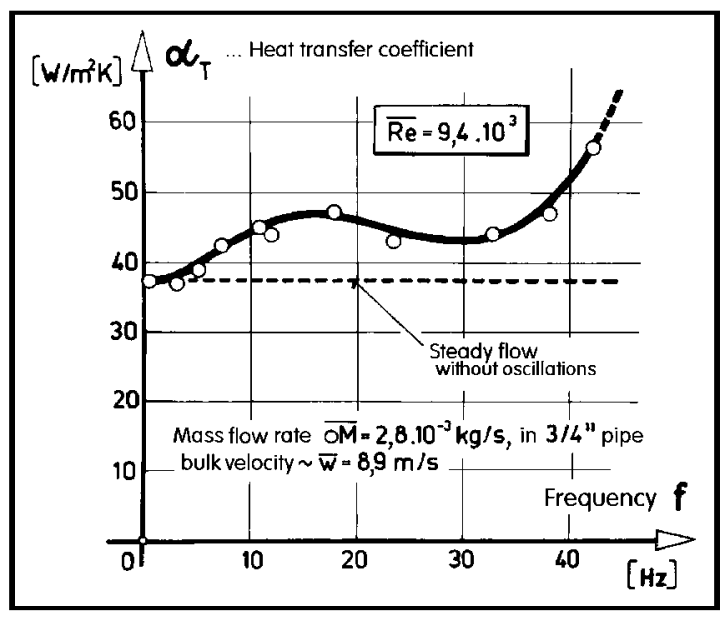

Fig 3 Heat transfer increase by fluidic oscillator obtained in experiments made together with student Mr. Marvan [8]. Conclusions: a) heat transfer increase is possible, b) the response to frequency variations is not monotonous but shows resonance peaks, c) time available for the student's diploma work expired before he could investigate the promising range of yet higher frequencies. 
simple heat exchanger itself. Moreover, although the heat transfer was indeed enhanced, the additional power input needed to drive the mechanical pulsator was always greater than what was gained by increased transfer in energy terms - as was perhaps typically demonstrated in ref. [4]. Consequently, the whole idea of pulsation was almost completely abandoned.

A solution proposed in 1970 s by the present author was to generate the pulsation in a much simpler way: by no-moving-part fluidic oscillators. These are essentially nothing more than just a special shaping of the jet generating nozzle. There are no moving components, no worn seals or bearings, no seizure damage, and no bolts, screws, and other components that could get loose. The fluidic oscillator may be maintenance-free and extremely reliable. The admittedly somewhat complex oscillator shapes are no more a problem for modern manufacturing methods like laser cutting or photoetching.

An early verification of the heat transfer enhancement by the use of a fluidic oscillator is described in [8], from which is taken Fig. 3. How this idea of pulsatory impinging jets with fluidic oscillators may be practically applied for the purposes thermal food-processing (baking or roasting) is shown in Figs. 1 and 2, taken from the author's Patent [5]. After almost two decades of absence of any interest in this patent (which expired in the meantime) the idea - together with interesting facts about the annular nozzles learned in the meantime [7, 35-37, 40, 44] - to improvement of microwave ovens as described in [6] in the project supported by Faraday Food Processing Activity, United Kingdom, Proof of concept fund 24063.

As is often the case, it was later discovered that the idea of circumventing the costly and energy consuming mechanical pulsator units was tackled by others. The selfexcited oscillation of a compressible fluid flow was demonstrated in [9] to increase the impingement flow heat transfer, actually without an oscillator as an additional device. The special shaping of the nozzle producing self-excited pulsation used there was invented earlier by Hill and Greene in 1977 [10]. Their "whistlernozzle" has a circumferential step ("collar") causing flow separation, the frequency of which was controlled by organ-pipe resonance in a constant-section channel in the nozzle. Another earlier development was the nozzle with an in-built oscillator of Narumanchi et al. [11]. The shape of their self-excited oscillation cavity was, however, complex and rather strange, the tortuous flow-paths inevitably leading to high hydraulic losses. Also an absence of any features for pressure recovery [12] certainly did not suggest efficiency - as was proved by the experiments. Thus these earlier attempts were just another dead branches of development.

\section{Experiment}

\subsection{Setup}

Being aware that hydraulic losses in the oscillator may negate whatever advantage is gained by the improved heat transfer, the present author in his experimental setup put an emphasis on a design with small dissipative losses.

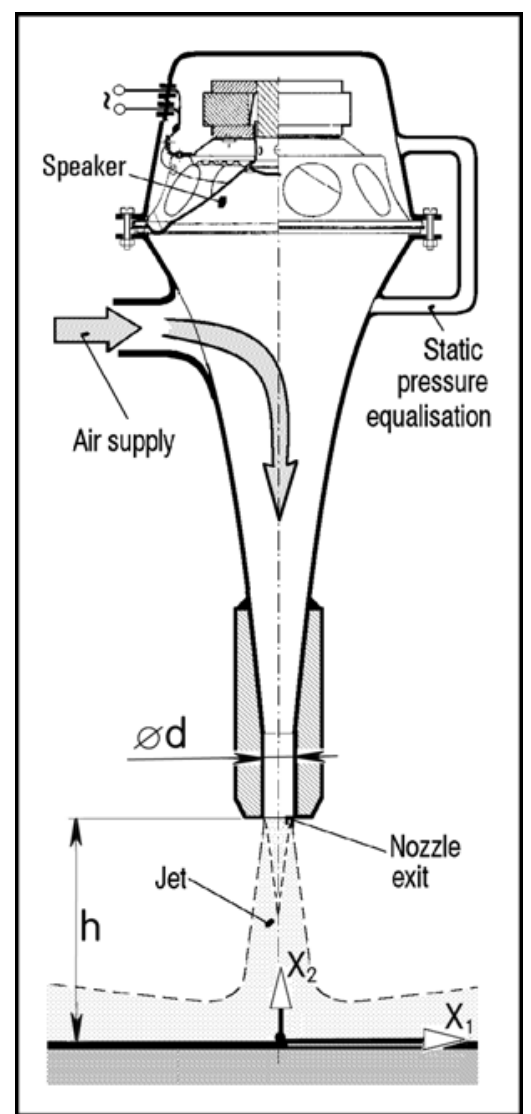

Fig 4 Author's set-up of the nozzle with loudspeaker-generated flow pulsation. This replaced the otherwise preferable fluidic oscillator because of independently adjustable frequency and flow rate. This nozzle was used in the experiments that led to the results presented in Fig. 8 .

His oscillator is based on the classical idea of a fluidic amplifier and de-stabilising feedback loop - as suggested above in Figs. 1 and 2. The amplifier is of the Coandaeffect bistable version so that the oscillation consists of alternating switching of the flow between the two attachment walls. Details of the amplifier are based on earlier experience with the author's successful geometry as it is described, e.g., in references [13] and [14].

Perhaps surprisingly, many of the author's laboratory investigations were actually made without a fluidic oscillator - replaced in its role by a loudspeaker driven by an electric signal, as shown in Fig.4. The reason was not a desertion from the idea of using fluidics. It was guided by ease of performing the measurements. It must be said that fluidic oscillators themselves still need a lot of detailed investigation and this complicates their use in experiments. A typical property of periodic processes in fluids in general is the (more or less) constant value of Strouhal number. This means that one of the essential variables of the tests, the oscillation frequency, is related to the supplied fluid flow rate. The two variables cannot be varied independently. This does not cause problems in the planned final application - but is inconvenient for laboratory investigations. The electrically driven version as presented in Fig. 4 avoids this inconvenience and makes possible independent adjustment and measurement of test conditions. 


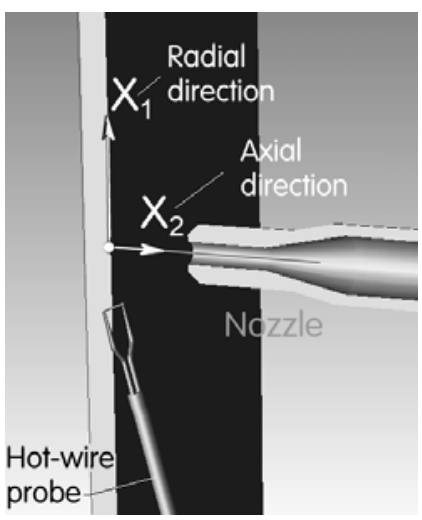

Fig 5 Anemometer measurements were made with the probe wire oriented tangentially - i.e. insensitive to the tangential velocity component.

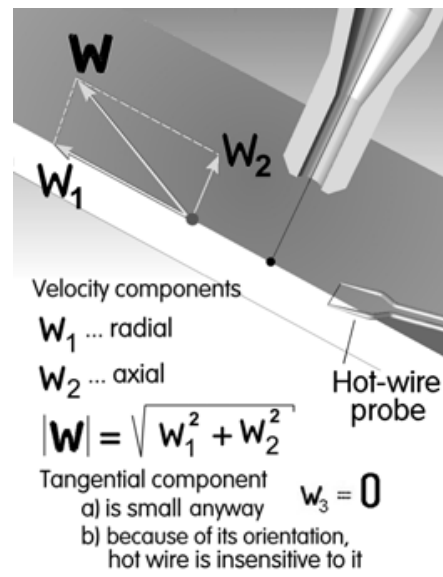

Fig 6 The velocity to which the probe positioned according to Fig. 5 responds by its output signal is the magnitude $|\boldsymbol{W}|$ of the velocity vector in the meridian plane.

The mentioned availability of the anemometric instrumentation led to using the anemometric probe in attempts at deeper understanding of the impinging jet flowfield. In particular, there was a belief that anemometry can and will answer the question of the offaxis secondary maxima of convective transfer [15] - and that they will be instrumental in investigating the response of the conduction layer to jet pulsation. Because of the single-channel version of the available anemometer, a single wire probe had to be used. This was not expected to be a problem with the flowfield that is axially symmetric - Fig. 5 . The tangential component of flow velocity could be neglected, Fig. 6, since (a) no tangential momentum was imparted intentionally to the air flow in the nozzle, and (b) the probe oriented tangentially is insensitive to a possible fluid motion in the tangential direction.

The measured absolute value of the velocity vector $\mathbf{w}$ $[\mathrm{m} / \mathrm{s}]$ cannot be resolved into the two components $\mathrm{w}_{1}$ and $\mathrm{w}_{2}$. On the other hand, the hot-wire anemometry (compared with Pitot probe used previously) has the advantage of very much wider frequency band - and the possibility to resolve, by simple electric signal processing, the velocities into the time-mean and fluctuating components

$$
\boldsymbol{W}=\overline{\boldsymbol{W}}+\boldsymbol{W}^{\prime \prime}
$$

- the latter, of course, varying in time so that its timemean value was zero. To characterise its magnitude, it is common in studies of turbulence to evaluate its RMS value, which in the case from Fig. 6 is

$$
|\widetilde{\mathbf{W}}|=\sqrt{\overline{w_{1}^{\prime \prime 2}+\overline{w_{2}^{\prime \prime 2}}}}
$$

The simple models of turbulence that could be used for numerical computations of this flowfield are based on the idea of isotropy of turbulent motions - the same fluctuation intensity in all three spatial directions

$$
\sqrt{\overline{w_{1}^{\prime \prime 2}}}=\sqrt{\overline{\overline{w_{2}^{\prime \prime 2}}}}=\sqrt{\overline{w_{3}^{\prime \prime 2}}}
$$

This is admittedly a rather drastic simplifying condition (the large ring-shaped or spiral turbulent vortices known to exist in jet flowfield certainly do posses a spatial orientation) but it brings the advantage of characterising the turbulence by a simple scalar quantity - the turbulent viscosity - in the computations. Using eq. (3), the theoretical value of the fluctuation intensity is

$$
|\widetilde{\mathbf{W}}|=\sqrt{3 \overline{w_{1}^{\prime \prime 2}}}
$$

The relative intensity of turbulence is judged by comparison of this quantity with the measured time-mean quantity

$$
|\overline{\mathbf{W}}|=\sqrt{\bar{w}_{1}^{2}+\bar{w}_{2}^{2}}
$$

Plotted on the vertical co-ordinate in the graphical presentation of experimental results is the ratio $|\widetilde{\mathbf{W}}| /|\overline{\mathbf{W}}|$ of the values computed from eqs. (4) and (5).

\subsection{Basic problem of anemometry}

The essential problem with the anemometric measurements are caused by the necessity of incorporating into the results the fluctuating velocity components which cannot be measured and their inclusion is dependent on unreliable assumptions. It is not only the case of the tangential component in eq. (40. Also not measured but evidently present and influencing the output of the hot-wire probe is the fluctuating component of the transversal velocity $\mathrm{w}_{2}$. Traditional approach to hot-wire anemometric measurements assumes the velocity fluctuations to be much smaller than the timemean velocity. This makes some approximations more or less acceptable. The assumption of small relative intensity of fluctuations is mentioned in all textbooks on hot-wire anemometry, but is generally neglected in practice - except that it is recognised that hot-wire anemometry should not be applied in the flows like the present impinging jet case. This explain the scarcity of data similar to those of the present author. The key problem is the necessity of linearisation of the probe signal - otherwise the data would be deteriorated by spectral dispersion associated with the non-linearities. Indeed, the dependence between the actually measured hot-wire resistance changes and the fluid velocity (King's law) is nonlinear. There is a widespread belief that the problem of nonlinearities is eliminated by performing calibration and subsequent signal linearisation, either by electronic hardware or in the software used for data processing. However, the linearisation of a flow with 
fluctuation is in the very basic principle an impossibility, as was demonstrated in [16].

Let us assume, for simplicity, the case of a parallel flow in the direction $X_{1}$. The probe responds to the instantaneous effective velocity $\mathrm{w}_{\text {eff }}$ normal to the wire axis:

$$
w_{\text {eff }}=\sqrt{\left(\bar{w}_{1}+w_{1}^{\prime \prime}\right)^{2}+w_{2}^{\prime \prime \prime}}
$$

(Note again the probe insensitivity to the third, tangential flow component $\mathrm{w}_{3}$, Fig. 6 ). In the standard approach the (small) transverse fluctuation $\mathrm{w}_{2}$ " in eq.(6) is neglected. Since the time-mean value of fluctuation is $\overline{w_{1}^{N \prime}}=0$, the measured quantity is the desirable

$$
\overline{w_{\text {eff }}}=\overline{w_{1}}
$$

In the outer parts of the impinging jet flows of the interest in this discussion, however, the fluctuation $\mathrm{w}_{2}$ " is not negligible. As a result, it is then inherently impossible to perform the linearisation correction, because according to eq. (6) it depends on the unknown $w_{2}$ ". Hinze, in a classical early analysis [17], still reproduced in textbooks, evaluated the magnitude of the resultant error by computations using the idealised case of an isotropic, normally distributed and normally correlated turbulence. The approach he used has led to series expansions, from which he took only the leading terms. For turbulence intensity value 0.2 obtained Hinze, an estimate of the error about $\sim 2 \%$. This may be quite acceptable as commensurable with other errors in the measurements. However, it is questionable how typical is the Hinze's assumed turbulence case. Moreover, if the transversal fluctuation cannot be neglected, it would be necessary to take into consideration the additional terms in Hinze's expansion - but this is out of question because the coefficients in the expansion actually defy evaluation.

The only known possibility how to evade these problems is to use the "flying hot wire", moved fast by a mechanism across the test space. This is a very demanding set-up, made by exceptional researchers for themselves $[32,33]$ and not offered by any professional supplier.

In view of these facts it is necessary to view the data presented as the experimental results in Figs. 7, 8, and 11. They are only of a qualitative character. Perhaps this is the reason why no similar anemometric investigations of impinging jets were found described in existing literature.

\subsection{Measurement results}

Measurements were performed with air as the working fluid. The nozzle (Fig. 4) had exit diameter $\mathrm{d}=3.85 \mathrm{~mm}$ - rather small, due to the limited output power of the loudspeaker and the requirement of significantly large pulsation amplitudes. The results discussed here were all obtained with the same time-mean nozzle exit velocity $\mathrm{w}_{\mathrm{e}}=29.5 \mathrm{~m} / \mathrm{s}$ - monitored by a manometer strongly damped to indicate the time-mean pressure difference between the nozzle chamber and the atmosphere: the indicated difference was maintained at $\Delta \mathrm{P}=500 \mathrm{~Pa}$. This ensured the Reynolds number value $\operatorname{Re}=7.1 \quad 10^{3}$, sufficiently large for a well developed turbulent character of the jet. To ensure powerful oscillation, the acoustic

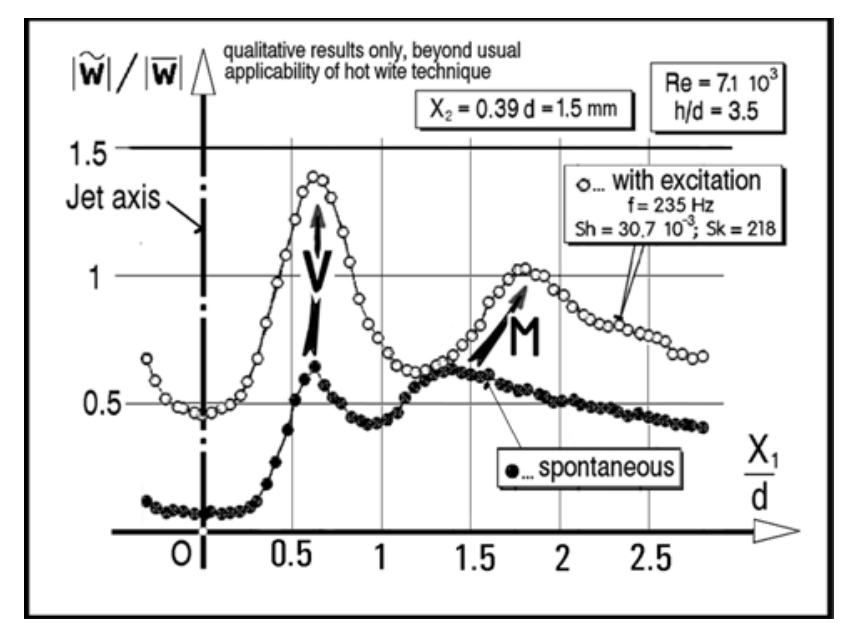

Fig 7 Author's anemometric data from ref. [19]: traversing the probe radially at a small vertical distance $X_{2}$ (cf. Fig. 4) above the wall exhibited two peaks $V$ and $M$ of turbulence intensity. They increased in magnitude with applied harmonic excitation.

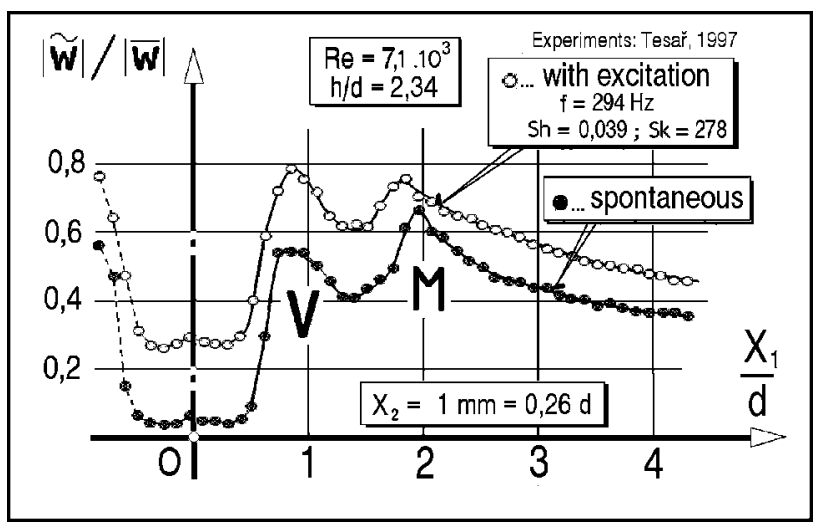

Fig. 8 The two peaks $V$ and $M$ of Fig. 7 were also found in another author's experiment with traversed probe - as shown here in a configuration with smaller distance $\mathrm{h}$ and different excitation frequency. Despite the same intensity of excitation, its effect is much weaker than in Fig. 8.

The hot wire probe for measurement of air flow velocity was 55P01, supplied by Dantec Dynamics Inc., with the wire of $5 \mathrm{~m}$ diameter and length $1.25 \mathrm{~mm}$. The constant temperature circuit of the anemometer kept the wire temperature at $200{ }^{\circ} \mathrm{C}$. The probe was traversed by a computer-controlled traverser between reading positions. At each position, the probe was kept in rest and 200000 measurements were taken of instantaneous velocity magnitude, at the sampling frequency $100 \mathrm{kHz}$. Statistical processing of these data produced both local time-mean absolute velocity value as well as the intensity of fluctuation at each point. There was, unfortunately, no precise positioning mechanism available for the $\mathrm{X}_{2}$ direction. Adjusting the distance of the probe wire above the impingement wall was based on visual estimations.

Results of the discussed impinging-jet experiments were presented in the research report [19], some of them reproduced here. In Figs. 8 and 9 there are the hot-wire measurement data obtained by radial traversing at a height $\mathrm{X}_{2}$ estimated to be $1.5 \mathrm{~mm}$, with the nozzle height $\mathrm{h}=3.5 \mathrm{~d}$ (Fig. 8) and $\mathrm{X}_{2}$ estimated $1 \mathrm{~mm}$ with the nozzle 


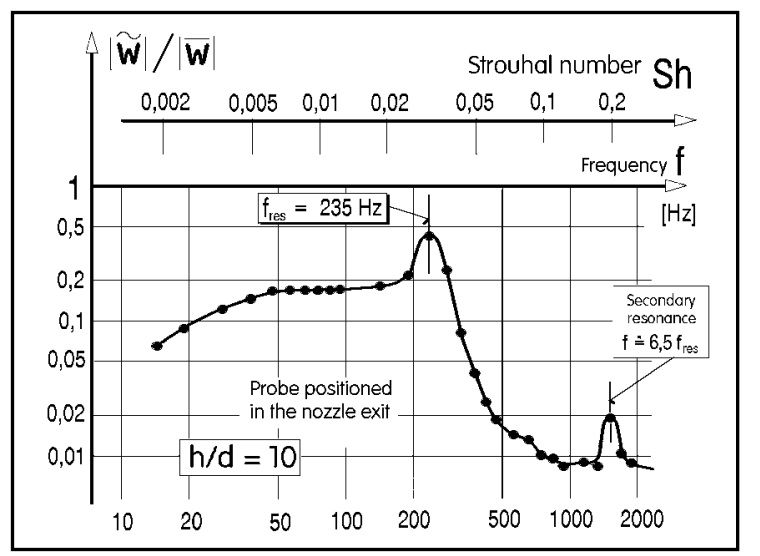

Fig 9 Frequency response of the actuator shown in Fig. 4 measured with the probe in the nozzle exit and large distance $h$. The main resonance peak at $\mathrm{f}_{\text {res }}=235 \mathrm{~Hz}$ was identified and this driving frequency was subsequently used in all investigations of the pulsatile impinging jets in [18].

exit at $\mathrm{h}=2.34 \mathrm{~d}$ (Fig. 9). It has to be emphasised again that the values of the fluctuation intensity plotted in this diagram are only qualitative - because at the level of fluctuation seen there is $100 \%$ and more, for which it is impossible to perform precise linearisation, as discussed above. in Sect. 2.2.

Nevertheless even these qualitative results are interesting. There are in Figs. 8 and 9 two data sets obtained either for the spontaneous turbulence or with the periodic excitation. Both distributions show immediately apparent local maxima. There is the more or less expected maximum $\mathrm{V}$ near the axis - and the not really expected second, off-axis maximum labelled M. The nozzle to wall distance was small enough for the making possible appearance of the off-axis secondary heat transfer maximum if the jet were heated. The discussed measurements were isothermal, but it is obvious that intensity of fluctuating motion corresponds to intensive convection transfer by eddies in the turbulence.

After the measurement run with steady supply air flow, the measurements were repeated with the loudspeaker connected to a source of harmonic electric signal. The amplitude of the excitation was evaluated by

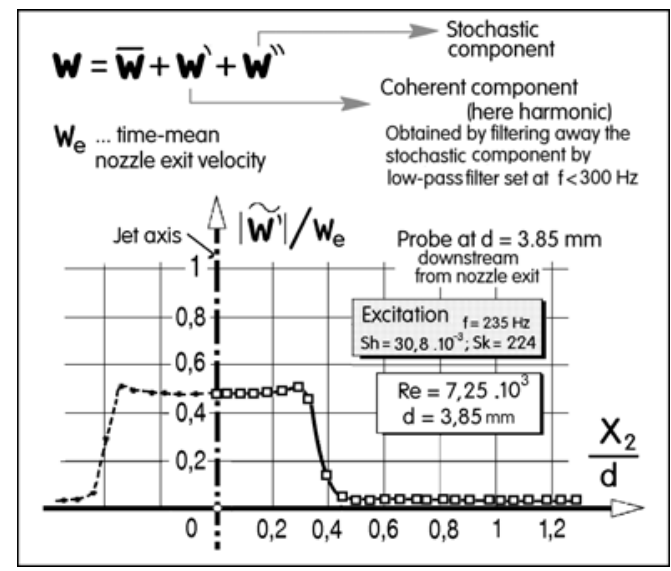

Fig. 10 Relative intensity of coherent oscillation in the "potential" core of the jet was measured to obtain information about the amplitude of the applied harmonic driving..

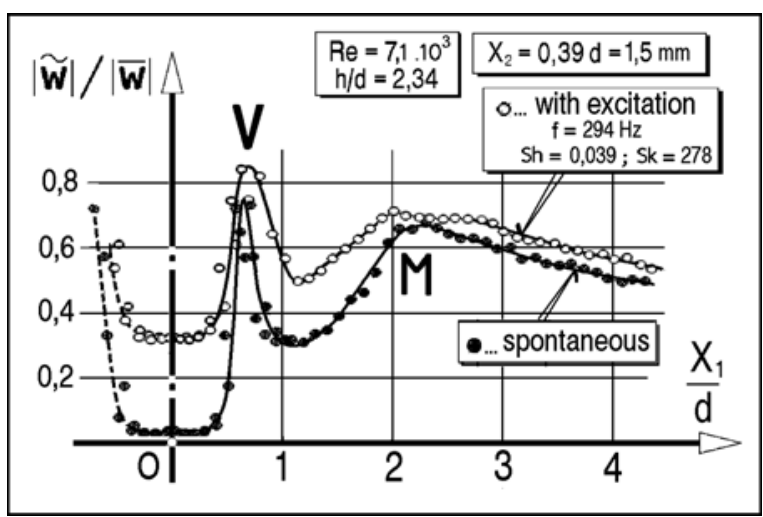

Fig. 11 Another author's experimental results - again revealing the peaks $\mathrm{V}$ and $\mathrm{M}$ as in Fig. 7, but here at smaller impingement wall distance $h$. Despite the same intensity of excitation, its effect is here much weaker than in Fig. 7.

measurements inside the constant-velocity core region of the jet, Fig. 10. To remove the influence of the natural turbulence, its taking into account was prevented by lowpass filtering of the anemometer signal. This was adjusted to remove whatever small-scale turbulence was there at frequency above $300 \mathrm{~Hz}$ (i.e. practically almost immediately above the $235 \mathrm{~Hz}$ driving signal). As shown in a typical Fig. 10, the root-mean-square value of the organised motion velocity magnitude inside the jet core was $48 \%$ of the time-mean velocity $\mathrm{w}_{\mathrm{e}}$ of the flow in the nozzle exit. The corresponding relative magnitude of the driving flow velocity amplitudes was 0.687 . The data obtained by the same traversing as in the natural turbulence are also shown in Figs. 8 and 9 and indicate that in the pulsating flow both maxima $\mathrm{V}$ and $\mathrm{M}$ remained present, only increased their magnitude. In Fig. 8 the pulsation moved the extra-axial maximum $M$ to a significantly larger radius.

In the measurements with smaller $\mathrm{h} / \mathrm{d}$, as here presented in Figs. 8 and 11, the harmonic driving did not result in such dramatic increase of measured fluctuation intensity as in Fig. 8. Also, there does not seem to be the radial displacement of the extra-axial maximum $\mathrm{M}$.

\section{Synergetic information from CFD}

\subsection{Need for additional data}

When the described hot-wire measurements were made, the author did not possess any other means for acquiring additional information about the investigated flowfield. He formulated a hypothesis according to which the measured local maxima $\mathrm{V}$ and $\mathrm{M}$ of relative fluctuation intensity were providing an explanation for the known off-axis local maxima of heat transfer as shown in Figs. 21,22 . However no additional information about $\mathrm{V}$ and $\mathrm{M}$ could be obtained. Publication of the hot-wire data beyond the brief description [19] at a local conference was not considered suitable because of their merely qualitative character - caused by the impossibility of evaluating the higher terms in the Hinze's expansion (discussed Sect. 2.2 above). 


\subsection{Numerical flowfield computations}

In several other author's recent research projects - e.g. in [21] - successful explanation of found experimental facts was obtained by synergetic approach: performing together with the laboratory measurements also parallel numerical flowfield computation. Computed results may provide information about details that are practically inaccessible by the probes. This success has led to trying a similar approach also in the present case of the two local maxima $\mathrm{V}$ and $\mathrm{M}$ in impinging jets.

The numerical solution was made in the domain the geometry of which is presented in Fig. 12. Because of the axial symmetry of the flowfield and absence of tangential flow, the computation could be made as twodimensional, in the meridian plane to save the solution time. Since preliminary computations did not show any interesting behaviour inside the large space of the nozzle (as shown in Fig. 4), only the small part of the nozzle near the exit was included into the computation domain. Inflow boundary condition (constant velocity in the steady flow case) was applied at the cross section located

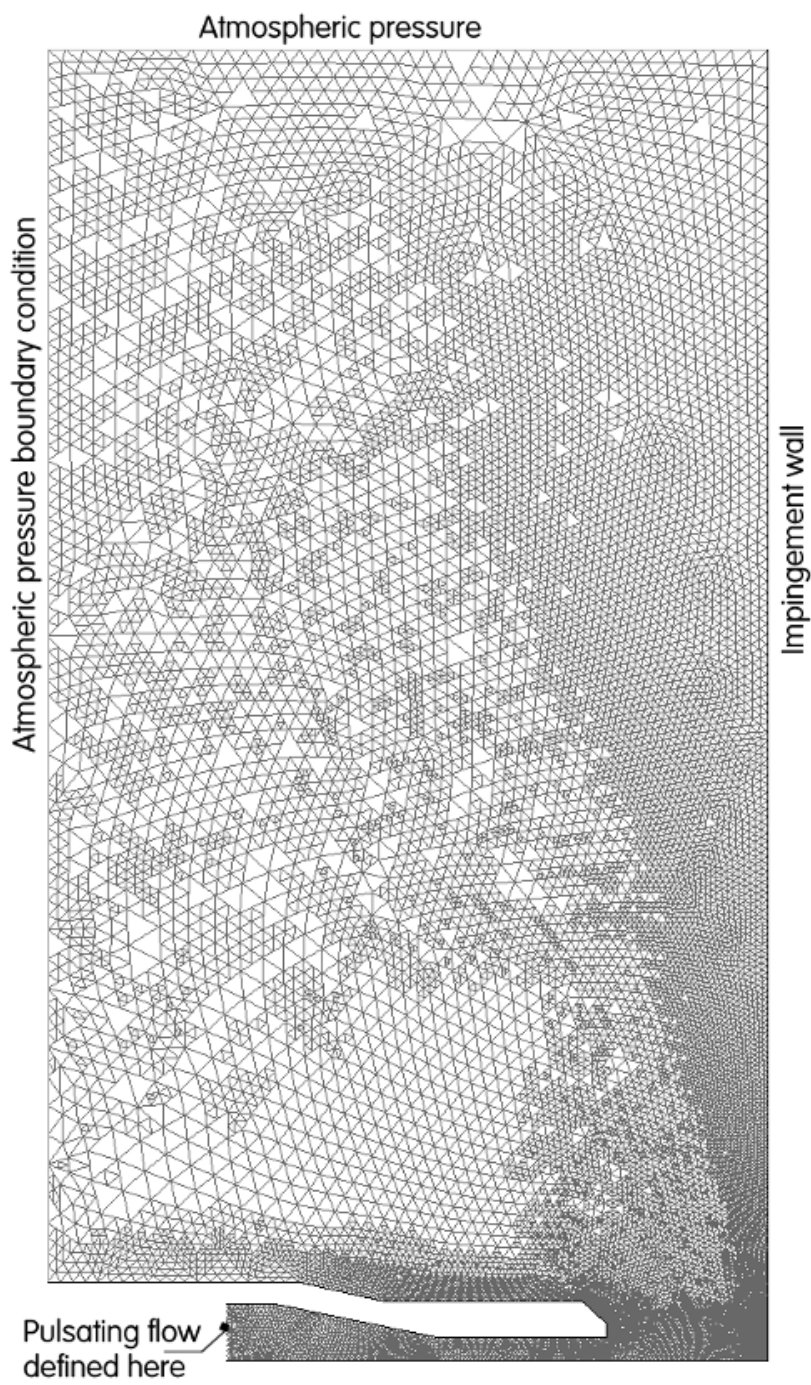

Fig. 12 The unstructured triangular discretisation grid used in the numerical flow computations. This is the final grid as it appeared after repeated adaptation by refinements in the regions with high gradient of velocity magnitude.

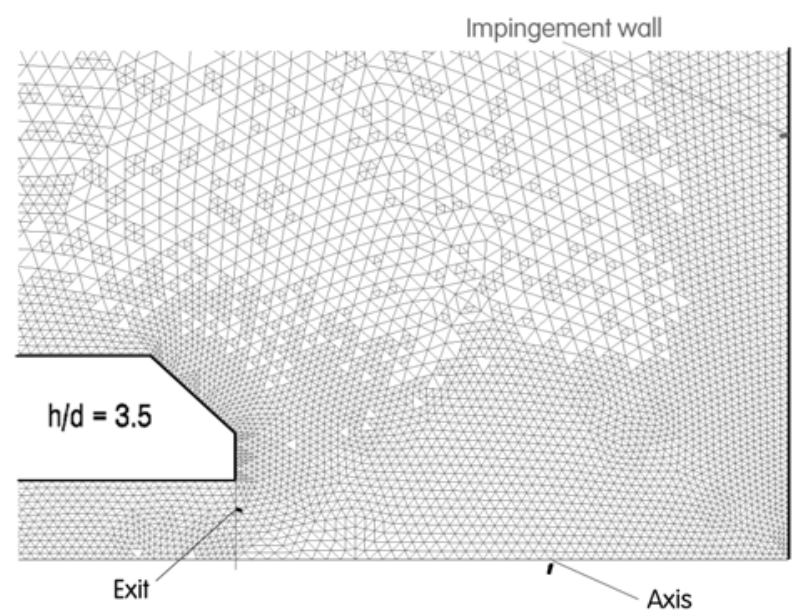

Fig. 13 The discretisation grid in the vicinity of the nozzle exit - where the refinement of the grid elements made the details indistinguishable in Fig. 12.

at 8.27 nozzle diameters $d$ upstream from the exit - thus retaining a part of the conical internal shape of the nozzle. The numerical solutions of the flowfield used the commercial software package FLUENT release 6.3.26. The governing equations were the standard Navier-Stokes equations solved by finite volumes discretisation. The domain was discretised by software GAMBIT 2.4.6. The used grid was unstructured triangular, adaptively refined during the computation. Typically, the computation was run with 29362 finite-volume cells. The criterion for application of the grid refinement was a pre-selected level of local gradient of velocity magnitude. Figures 12 and 13 (the latter a detail of the small size grid near the nozzle exit) show a typical mesh in the symmetry plane after the refinement. Turbulence was modelled by standard twoequation (k-epsilon) model with the low turbulence Reynolds number behaviour resolved using the RNG approach, which was in previous authors' computations found capable of handling the low turbulence Reynolds number behaviour.

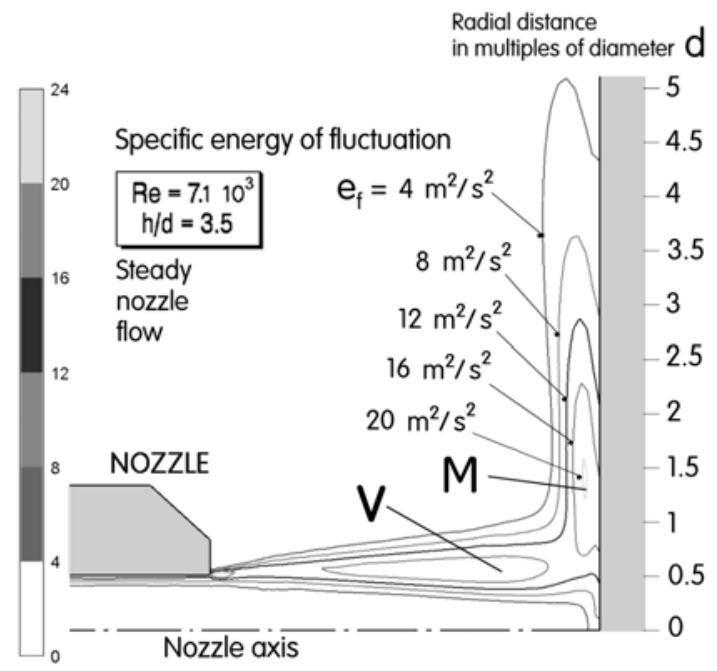

Fig. 14 Steady-flow computation results: spatial distribution of the specific energy of fluctuation (in this case a spontaneously generated turbulence) shows two local extremes that may be interpreted as the maxima $\mathrm{V}$ and $\mathrm{M}$. 


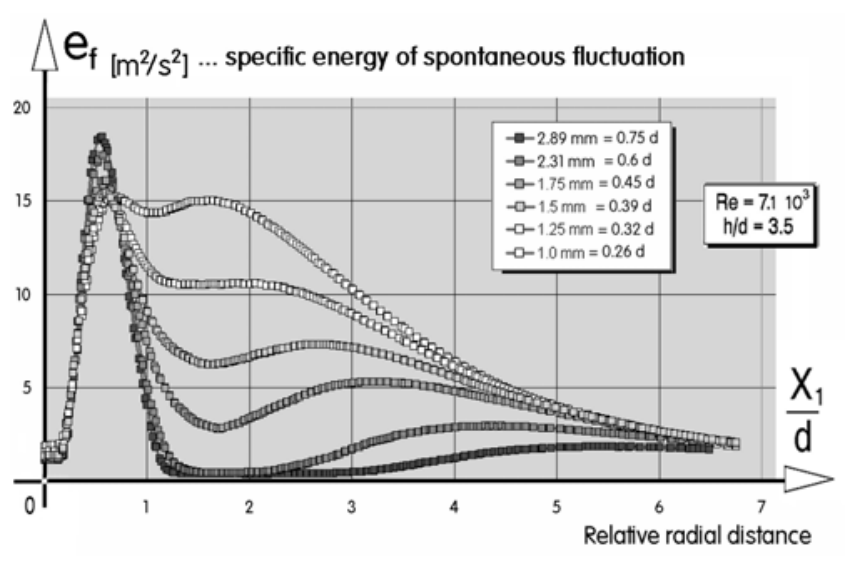

Fig. 15 Steady-flow computations: radial distribution of specific energy of (spontaneous) fluctuation along six different radial lines at different height above the impingement surface.

A typical result of the computations with steady supply flow is presented in Fig. 14 - in the form of the distribution of specific values of energy of turbulent fluctuations $e_{f}\left[\mathrm{~m}^{2} / \mathrm{s}^{2}\right]$. Note that in isotropic turbulence eq. (3) - the fluctuation intensity eq.(4) is

$$
|\widetilde{w}|=\sqrt{2 e_{1}}
$$

In the initial computations of the steady-flow case, the fluctuations are, of course, those of spontaneous turbulence. The Reynolds number $\mathrm{Re}$ as well as the configuration $\mathrm{h} / \mathrm{d}$ were those indicated above in Fig. 8. As expected, the fluctuation energy $e_{f}$ has zero value inside the nozzle and also in the jet core downstream from the exit. Quite high values are in the mixing layer outside the core - in the location indicated in Fig. 14 as probably corresponding to the local extreme $\mathrm{V}$ of Fig. 8. This distribution of energy in the outer mixing layer corresponds to those known in non-impinging jets. The impingement, however, generates another local maximum: near the wall and further away from the

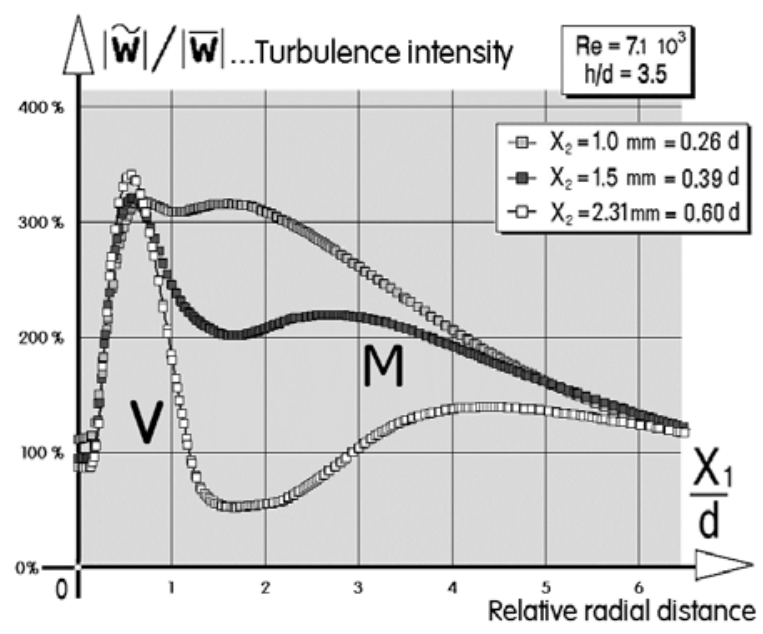

Fig. 16 The same computation results as in the previous Fig. 15 converted into the radial distributions of relative intensity of turbulence. Because of being related to the decreasing local time-mean velocity, the two maxima $\mathrm{V}$ and $\mathrm{M}$ are here of comparable magnitude. nozzle axis. This is in Fig. 14 labelled M. To what degree this distribution corresponds to the experimental results presented in Fig. 8 may be judged by considering the distribution of the energy $e_{f}$ along a near-wall radial line (in Fig. 8 this is the line at $\mathrm{X}_{2}$ estimated, perhaps not exactly, to be $1.5 \mathrm{~mm}$ ). The computed values - not only for a single height above the impingement wall but for a number of various small heights - are plotted in Fig. 15. Indeed, there is the near-axis maximum followed in the radial direction by a local decrease - and then further downstream a local increase. By adopting eq. (6) it is possible to evaluate from Fig. 15 the radial distributions of turbulence intensity. The computed values, directly comparable with the experimental data in Fig. 8, are is presented in the next Fig. 16. Because the magnitudes of fluctuation are divided by the local time-mean velocity, which rather rapidly decreases in the radial direction, the computed distributions in Fig. 16 possess a higher value of the maximum $\mathrm{M}$, commensurable with $\mathrm{V}$. In the comparison with Fig. 8, it is necessary to take into consideration all the problems mentioned above. On the side of the experiment it is the imprecise (only visually estimated) positioning of the hot wire probe and the impossibility of its linearisation (typically, in Fig. 16, the turbulence intensity values are $\sim 300 \%$ while the limit for application of Hinze's analysis is $20 \%$ ). On the side of the numerical solutions there are all the approximations admitted in the model of turbulence (in particular the isotropy and very rough modifications applied to the turbulence model for flows with low Reynolds numbers of turbulence). Thus the qualitative agreements, such as the presence of the maxima $V$ and $M$ found in both approaches, may be described as successful.

\subsection{Periodic flow}

In the next step of investigations, the numerical flowfield solutions were extended to the case of periodic unsteady supply flow. The governing equation in this extension were incorporating the unsteady term and the solutions were made by waiting for convergence at each of individual time steps. The integration with respect to time was 1st-order implicit. There were 80 time steps in each pulsation period, so that the time step size (which was fixed) at the $\mathrm{f}=235 \mathrm{~Hz}$ driving frequency was 53.191 $10^{-6} \mathrm{~s}$. The solutions progressed over several periods until the character of time histories became stabilised (by damping of the initial deviations which were caused by initial conditions - which were necessarily different from the solution, initially not known). The boundary conditions were as shown in Fig. 12, i.e. with harmonically varying inlet velocity inside the nozzle superimposed on the steady value. The Reynolds number indicated in the pictures was evaluated from the steady component.. Apart from the relative amplitude 0.687 of the sinusoidal component, two other alternating supply flow component magnitudes were also used, 0.848 (larger) and 0.424 (smaller than the experiment value) The two-equation model of turbulence was retained. It is therefore necessary to emphasise the Reynolds-averaged character of the solution. The presence of the vortical 
Velocity boundary condition at $\mathrm{S}$

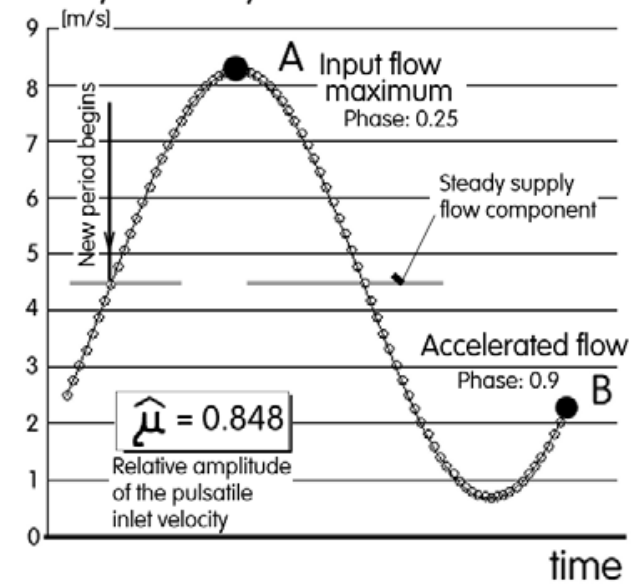

Fig. 17 The pulsatile flow applied in the unsteady computations at the inlet flow boundary condition location. This is one of the computation cases with relative amplitude of pulsation higher than in the experiment. Individual data points correspond to the 80 solution time steps. The two points A and $\mathrm{B}$ correspond to the phase positions of the solutions presented in Figs. 18 and 18.

motions (eddies) in the flowfield and their spatial transport was modelled by the standard gradient-diffusion terms in the governing equations. Thus the computed flowfield does not contain recognisable individual eddies, only the phase-averaged mean flow. There is in Fig. 17 a diagram in which, as a function of time, are the data points in which were computed instantaneous velocity values in the flowfield (the velocity on the vertical axis is the driving flow value in the boundary condition position indicated in Fig. 12). The following Figs. 18 and 19 present the distributions of the specific fluctuation energy $e_{f}$ in the vicinity of the nozzle, similar to the steady-flow case in Fig. 14 above. Now, in the unsteady case, there is a different flowfield computed for each time

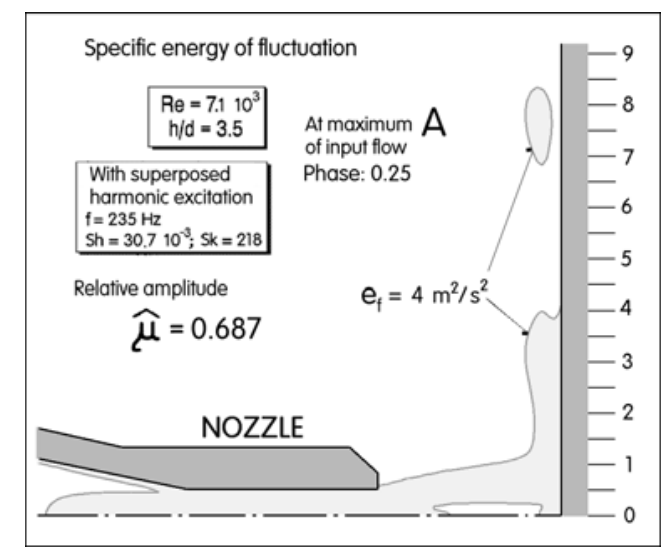

Fig. 18 Instantaneous spatial distribution of specific energy of unsteady motions computed for the instant at which the input pulsatile flow component reaches its maximum (Fig. 17). The boundaries delimiting the gray-colour region of high fluctuation intensity show there is an area that moves away from the impingement. The relative input velocity amplitude corresponds to the experimental case in Fig. 10. step - and of them Figs. 19 and 19 show the instantaneous distribution of the energy of fluctuations $e_{f}$ at the instants $A$ and $B$ in Fig. 17. The energy of fluctuations is here not just the energy of turbulence, but also represents the specific energy of the periodic motion. This, of course, becomes gradually converted into turbulence, but in the region of interest still retains the non-stochastic phase-averaged character, recognisable in the solutions as waves propagating from the nozzle.

Thus in the two examples shown in Figs. 18 and 19 these waves have the character of convective motion carrying the regions recognisable by their higher local energy of unsteady motion away in radial direction. .In Fig. 18 a small area of higher fluctuations has already reached the distance of $\sim 8$ diameters $\mathrm{d}$. In another case presented in Fig. 20 a similar area (for slightly different conditions) is shown at a phase in which it has moved to radial distance equal to 6 nozzle exit

Obviously, the transport of energy from these wavelike motion into the modelled stochastic turbulence is not rapid enough. Thus the waves of the organised-motion packets are also clearly recognisable in the radial distribution shown in Fig. 19 computed for 8 different instants equidistantly distributed over one pulsation period. The waves are seen to move radially away from the nozzle axis. Because of this rather slow conversion of organised motions into the chaotic turbulence, the timemean distribution, obtained in Fig. 20 by averaging of computation results obtained in individual time steps, retains essentially its distribution for the steady flow as shown in Fig. 16. The signal processing of the hot-wire measurements did not discriminate between the chaotic and organised motions and, as a result, the values in Fig. 8 shows the sum of both chaotic and organised energy components.

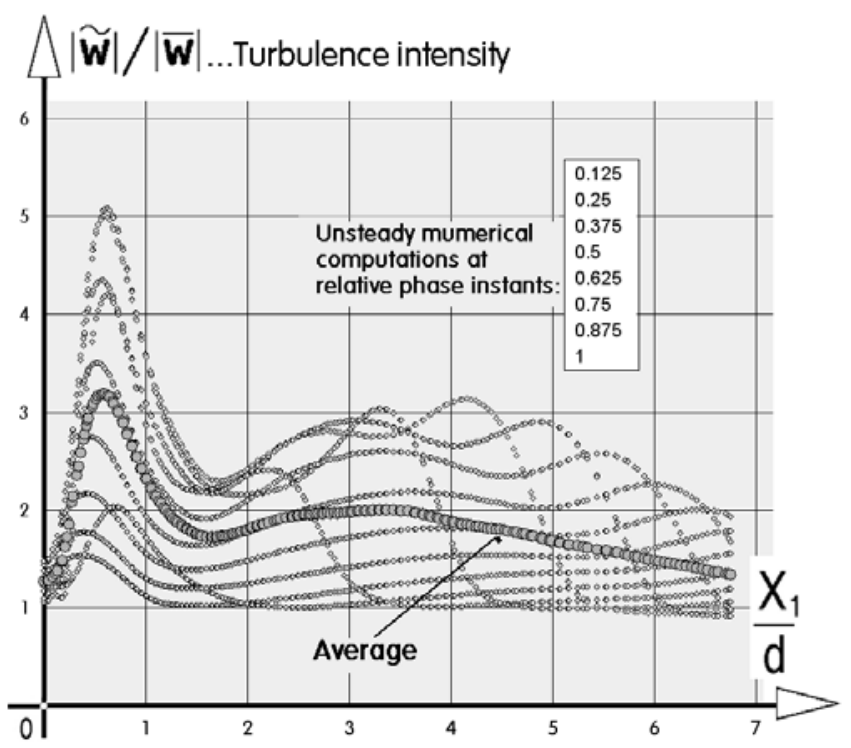

Fig. 19 Computed distributions of intensity of unsteady motions along a radial line at $1.5 \mathrm{~mm}$ above the impingement plane. Pulsating input flow generates waves of unsteadiness what move radially away from the impingement. Only eight representative phases (not all 80 time steps, but only those separated mutually by 10 time steps) were selected for presentation. 


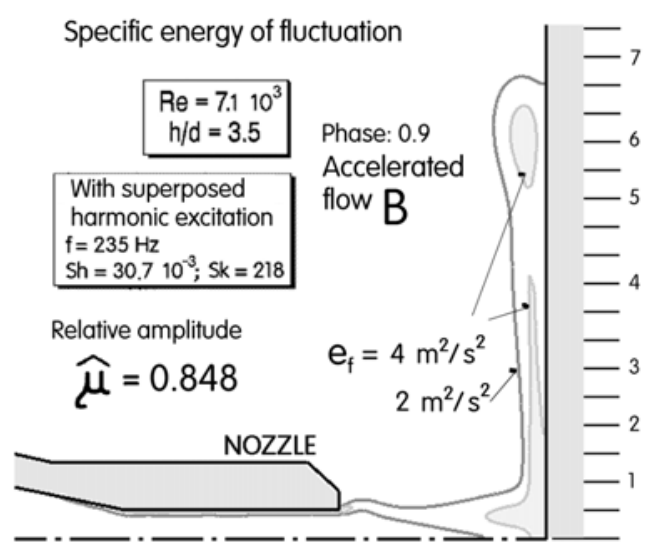

Fig. 20 Computed distribution of specific energy of unsteady motions in the regime of accelerated input flow (case B in Fig. 17). In this computed solution, the relative input velocity amplitude is slightly higher than the experimental case in Fig. 10.

Characteristically, the distributions like those in the diagrams presented in Figs. 7, 8, and 11 show an increase due to the pulsation also on the jet axis location, where it is only the effect of organised motion.

\section{Interpretation}

Apart from the authors' experimental and numerical solution results, the explanation and interpretation of the observed $V$ and $M$ maxima should take into consideration also all available information from literature. Practically all such information is deduced indirectly, not from measurements and analyses of the flowfield but from evaluations of heat- (and, in much lesser degree, mass-) transfer conditions on the impingement surface. The heat transport data are usually converted into the radial distributions of Nusselt number. At small relative nozzle distances $\mathrm{h} / \mathrm{d}$, as presented in the two representative examples Figs. 21and 22, from refs. $[23,24]$, the experiments exhibit local maxima of the Nusselt number that seem to be reasonably identified with the present authors' maximum values at $\mathrm{V}$ and $\mathrm{M}$.

What direct information is now available from the two approaches discussed in the present paper? Of

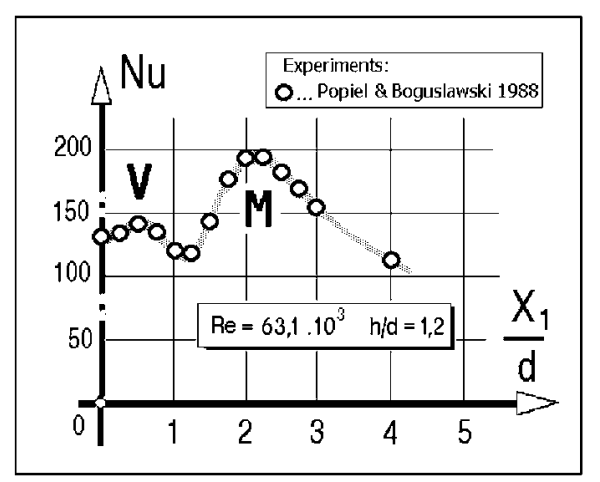

Fig. 21 Existence of the two local maxima $\mathrm{V}$ and $\mathrm{M}$ was also found in heat transfer measurements - here by authors of ref. [ 23].

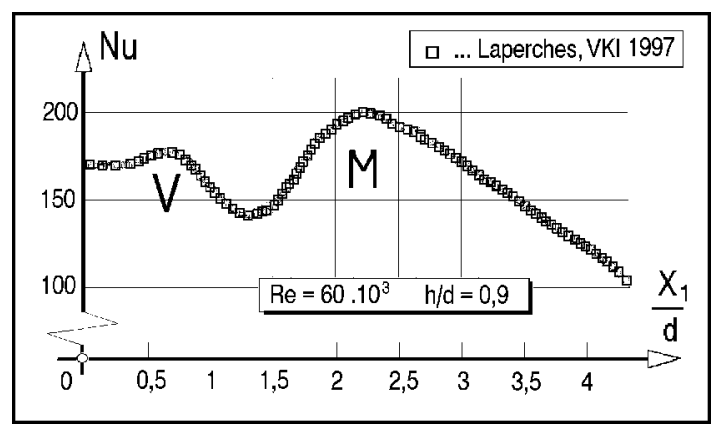

Fig. 22 Another demonstration of the presence of maxima $V$ and $\mathrm{M}$ by measurements of Nusselt number distributions - from [24].

course, it is necessary in both cases to consider their limitations (as they were already mentioned above), summarised as follows:

(a) Traversing by the single hot wire in this case can produce only qualitative data because of the impossibility of evaluating the higher terms in Hinze's expansion. Nevertheless, the results are a sufficient proof of velocity fluctuation intensity reaching at the $\mathrm{V}$ and $\mathrm{M}$ positions the local maxima.

(b) Numerical solution based on the used isotropic model of fluctuation and the impossibility to follow individual eddies because of the Reynolds-type averaging at a certain time-step phase, though far from perfect, shows the peaks that may be associated with those in the experimental results.

Thus despite their individual shortcomings, both approaches together suffice for quite reliable identification of the local maxima of unsteady motions energy at the two $V$ and $M$ positions. The intensity of convective transport demonstrated to be present at the impingement wall below these regions of intensive

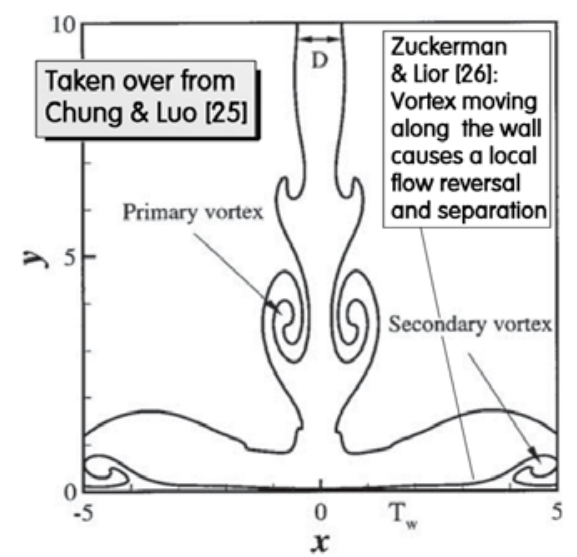

Fig. 23 This picture taken from ref. [25] shows a typical present-day explanations in current literature of the existence of the secondary maximum $M$. In fact the results in [25] were obtained at laminar-flow Reynolds number $\operatorname{Re}=500$ and at the relative nozzle height h/d (cf. Fig. 4) too large for presence of standard secondary maxima - so that the validity of this demonstration is somewhat doubtful. 
unsteady motion of the eddies is a sufficient document of the fact that the convective transport is due to the turbulence reaching to the wall.

In current literature, the existence of the off-axis maximum of convective transport intensity is based on observations, in visualised flow, of the so-called "secondary" vortices, as shown in Fig. 23 - taken directly from [25] with the added note taken from [26]. These conjectures are actually somewhat doubtful as they rely upon the separation and flow reversal that were not observed.

The idea established on the basis of the results from the present paper, in agreement with the early hypothesis formulated in ref. [15] is the following: the local extremes of transport intensity are due to the action of the fluctuating motion carried in a wave-like manner by the basic flow. This explanation, by manifestations of the convective origin of the maximum $M$ thus seems to be more reasonable.

\section{Conclusions}

The information obtained from the present research project may be summarised as follows:

(1) It is demonstrated that the two local extremes found by the author in impinging jet flow already in 1997 and labelled $\mathrm{V}$ and $\mathrm{M}$ are a proven fact.

(2) The two extremes remain in existence even in the externally excited pulsating impinging flows.

(3) The extremes have their initial origin in the flow instability in the mixing layer on the outer boundary of the jet outside of its core. This instability results in vortical motion that is convected along the outer layer of the jet flow.

(4) The higher local intensity of fluctuation in the extreme $\mathrm{M}$ - in the outer layer of the radial wall jet generated by the impact - is transferred towards the impingement wall below. This is an important fact from the application (heat and/or mass transfer) point of view.

(5) Rather low-level experimentation (a single-channel single-wire anemometer without adaptation for high fluctuation intensity) and also low-level (isotropic, averaged) numerical flowfield solutions were found sufficient for a convincing explanation of the processes taking place of the local maximum $\mathrm{M}$.

(6) In pulsated impinging jet, transport of energy from the organised pulsation into the chaotic turbulent transport is not rapid (at least at Reynolds numbers of the order $10^{4}$ ). The fluctuation is not dissipated but is carried away radially in a wave-like manner.

\section{Acknowledgements}

Author gratefully acknowledges the support by research grant 13-23046S obtained from GAČR - the Czech Science Foundation.

\section{References}

1. E.P. Dyban, A.I.Mazur, Convective heat transfer in jet-type flow past bodies (in Russian, Naukova dumka, Kiev, 1982)

2. G.G. Stokes, Transactions of the Cambridge Philosophical Society, IX, 8 (1851)

3. R. C. Martinelli, L.M.K. Boelter, Proc. of $5^{\text {th }}$ International Congress of Applied Mechanics, (Cambridge, Mass., USA, 1938)

4. W. Linke, W. Hufschmidt, Chemie-Ingenieur Technik 30, 159 (1958)

5. V. Tesař, P. Topinka, Czechoslovak Certificate of Authorship Nr. 263 898, filed December 1987

6. V. Tesař, Chemical Engineering Research and Design 87, 181 (2009)

7. V. Tesař, Z. Trávníček, Journal of Visualization,. 8, $91(2005)$

8. V. Tesař, L. Marvan L., Acta polytechnica-Práce ČVUT v Praze, Nr. 4, Series II, Praha 1989

9. R.H. Page, P.S. Chinnock, J. Sezeed-Yagoobi, Journal of Thermophysics and Heat Transfer 10, 380 (1996)

10. W.G. Hill, P. R. Greene, Journal of Fluids Engineering 99, 520 (1977)

11. S. Narumanchi, et al., Single-phase Self-Oscillating Jets for Enhanced Heat Transfer ( Conference paper SemiTherm 2008, San Jose, Calif.)

12. V. Tesař V., Sensors and Actuators A: Physical 152, 182 (2009)

13. V. Tesař, C.-H. Hung, W. B. J. Zimmerman, Sensors and Actuators A: Physical, 125, 159 (2006)

14. V. Tesar, H.C.H. Bandalusena, Experiments in Fluids 50, 1225 (2010)

15. V. Tesař V., The Problem of Off-Axis TransferEffect Extremes in Impinging Jets ( Proc. of XVIIth international scientific conference, 187, Herlany, Slovakia, June 1998)

16. E.J. Nielsen, et al., AIAA Journal 1, . 1210 (1963)

17. J. O. Hinze, Turbulence (McGraw-Hill Book Company, New York, 1975)

18. V. Tesař V., Research report Z-0149/97, ČVUT Praha, September 1997

19. V. Tesař V., Damping of oscillations in the near-wall layer of impinging jet flows (Proceedings of WORKSHOP 98, the 7th University-Wide Seminar, p.. 535, ČVUT Praha, February 1998)

20. V. Tesař, Excited Axisymmetric Impinging Flows (Proc. of Conf. "Engineering Mechanics '98", Vol. 4, p. 757, Svratka, Czech Republic, May 1998)

21. V. Tesař, Chemical Engineering Research and Design, 89, 436 (2011)

22. V. Tesař, Sensors and Actuators A - Physical, 179, $211(2012)$

23. C. O. Popiel, L. Boguslawski, Effect of flow structure on the heat or mass transfer on a flat plate in impinging round jet (Proc. of 2nd UK National Conf. on Heat Transfer, Univ. of Strathclyde, vol. 1, p. 663,1988$)$

24. J.-M. Buchlin, M. Laperches, Detailed investigation of aerothermal behaviour of confined impinging jet (Proc. of Conf. QUIRT Quantitative Infrared Thermography, p. 258, Lodz, Poland, 1998) 
25. R. Gardon, J. C. Akfirat, Int. Journal of Heat and Mass Transfer, 8, 1261 (1965)

26. G. M. Carlomagno, G. Cardone, Experiments in Fluids, 49, 1187 (2010)

27. V.Tesar, Z. Randa, Processing information obtained from thermochromic liquid crystals (Proc. of Conf. 'Experimental Fluid Mechanics 2007', Liberec, November 2007)

28. Y.M. Chung, K. H. Luo, Journal of Heat Transfer 124, 1039 (2002)

29. N. Zuckerman, N. Lior, Advancers in Heat Transfer 39, 565 (2006)

30. J. M. Buchlin, Journal of Applied Fluid Mechanics, . 4, 137 (2011)

31. Z. Trávníček, T. Hyhlík, F. Maršík, Synthetic jet impingement heat/mass transfer (Proc. of 16th Internat. Symp. on Transport Phenomena, Prague, 2005)

32. A. M. Al-Garni, Flow Measurement and Instrumentation 18, 95 (2007)

33 T. Persoons, A. Hoefnages, E. Van den Bulck, Experiments in Fluids 40, 555 (2006)

34. V. Tesař, J. Barker, Journal of Visualisation 5, 301 (2002)

35. Z. Trávníček, V. Tesař, International Journal of Heat and Mass Transfer 46, 3291 (2003)

36. Z. Trávníček, V. Tesař, International Journal of Heat and Mass Transfer 47, 2329 (2004)

37. V. Tesař, Z. Trávníček, Chemical Engineering Journal 144, 312 (2008)

39. Z. Trávníček, V. Tesař, Experimental Thermal and Fluid Science, 44, 565 (2013)

40. V. Tesař, M. Jílek, Z. Randa, Wall Pressure Distributions Under Impinging Annular Jets (Proc. of $20^{\text {th }}$ Internat. Conf., Kouty nad Desnou, 2001)

41. V. Tesař, Z. Trávníček, Pulsating and Synthetic Impinging Jets for High Heat and Mass Transfer Rates (Proc. of ESDA ýth Biennial ASME Cong., Manchester 2004)

42. V. Tesař, Conditions on the Wall under a Pair of Phase-Shifted, Impinging Hybrid-Synthetic Annular Jets (Proc. of ISFV12, Göttingen, 2006)

43. Z. Trávníček et al., Bifurcated and Helical Impinging Jet Controlled by Azimuthally Arranged Synthetic Jet (Proc. of HEFAT2007, Sun City, South Africa, 2007)

44. Z. Trávníček, V. Tesař Synthetic Jets used to Control Spreading of an Annular Impinging Jet (Proc. of ISFV13 - 13th Intern. Symp. on Flow Visualization and FLUVISU12 - 12th French Congr. on Visualization in Fluid Mechanics, Nice, France, 2008)

45. V. Tesař, J. Kordík, Sensors and Actuators APhysical, 191, 24 (2012)

43. Z. Trávníček et al., International Journal of Heat and Mass Transfer 55, 1279 (2012) 